\title{
Approximate Euler-Lagrange quadratic mappings
}

\author{
Hark-Mahn Kim, Juri Lee* and Eunyoung Son
}

\author{
* Correspondence: \\ annans@hanmail.net \\ Department of Mathematics, \\ Chungnam National University, 79 \\ Daehangno, Yuseong-gu, Daejeon \\ 305-764, Korea
}

\author{
Abstract \\ For any fixed integer $k$ with $k \neq 0,1$, we prove the Hyers-Ulam stability of an Euler- \\ Lagrange-type quadratic functional equation$$
f(k x+y)+f(k x-y)=k f(x+y)+k f(x-y)+2 k(k-1) f(x)-2(k-1) f(y)
$$ \\ in normed spaces and in non-Archimedean normed spaces.
}

\section{Introduction}

The problem of stability of functional equations was originally stated by Ulam [1]. In 1941, Hyers [2] gave an affirmative answer to Ulam's problem for the case of approximate additive mappings on Banach spaces. In 1950, Aoki discussed the Hyers-Ulam stability theorem in [3]. His result was further generalized and rediscovered by Rassias [4] in 1978. The stability problem for functional equation has extensively been investigated by a number of mathematicians [5-9].

The quadratic function $f(x)=c x^{2}$ satisfies the functional equation

$$
f(x+y)+f(x-y)=2 f(x)+2 f(y)
$$

and therefore Equation (1) is called the quadratic functional equation. Every solution of Equation (1) is said to be a quadratic mapping. The Hyers-Ulam stability theorem for the quadratic functional equation (1) was by Skof [9] for the functions $f: E_{1} \rightarrow E_{2}$ where $E_{1}$ is a normed space and $E_{2}$ is a Banach space. The result of Skof is still true if the relevant domain $E_{1}$ is replaced by an Abelian group and this was dealt with by Cholewa [10]. Czerwik [11] proved the Hyers-Ulam stability of the quadratic functional equation (1). This result was further generalized by Rassias [12], Borelli and Forti [13]. During the last three decades, a number of papers and research monographs have been published on various generalizations and applications of the Hyers-Ulam stability of several functional equations, and there are many interesting results concerning this problem [14-20]. In particular, Rassias investigated the Hyers-Ulam stability for the relative Euler-Lagrange functional equation

$$
f(a x+b y)+f(b x-a y)=\left(a^{2}+b^{2}\right)[f(x)+f(y)]
$$

in [21-23].

In 2008, Ravi et al. [24] investigated the Hyers-Ulam stability of a quadratic functional equation

$$
f(2 x+y)+f(2 x-y)=2 f(x+y)+2 f(x-y)+4 f(x)-2 f(y) .
$$

(c) 2012 Kim et al; licensee Springer. This is an Open Access article distributed under the terms of the Creative Commons Attribution License (http://creativecommons.org/licenses/by/2.0), which permits unrestricted use, distribution, and reproduction in any medium, provided the original work is properly cited. 
In this article, we generalize the functional equation (3) to a more general form

$$
f(k x+y)+f(k x-y)=k f(x+y)+k f(x-y)+2 k(k-1) f(x)-2(k-1) f(y)
$$

and investigate the Hyers-Ulam stability of the equation for any fixed integer $k$ with $k \neq 0,1$. As results, we improve the generalized stability results given in [24] in normed spaces and in non-Archimedean normed spaces.

\section{General solution of (4)}

First of all, if $k=-1$ in Equation (4), then it is easy to see that

$$
f(-x+y)+f(-x-y)=-f(x+y)-f(x-y)+4 f(x)+4 f(y)
$$

is equivalent to Equation (1), and so the solution of Equation (4) with $k=-1$ is a quadratic mapping. Thus, we consider general solutions of Equation (4) for any fixed integer $k$ with $|k|>1$ in the following theorem. The following lemma can be found in [25-27].

Lemma 2.1. A mapping $f: X \rightarrow Y$ between linear spaces satisfies the functional equation

$$
f(2 x+y)+f(2 x-y)=4[f(x+y)+f(x-y)]+2[f(2 x)-4 f(x)]-6 f(y)
$$

if and only if $f$ is quadratic and quartic.

Theorem 2.2. A mapping $f: X \rightarrow Y$ between linear spaces satisfies the functional equation (4) with $|k|>1$ if and only if $f$ is quadratic.

Proof. Let $f$ be a solution of Equation (4). Letting $x=y=0$ in (4), we have $f(0)=0$. Putting $y=0$ in (4), we get $f(k x)=k^{2} f(x)$. Putting $x=0$ in (4), we get $f(-y)=f(y)$. Thus, the mapping $f$ is even. Therefore, it suffices to prove that if a mapping $f$ satisfies Equation (4) for any fixed integer $k$ with $|k|>1$, then $f$ is quadratic. Now, replacing $y$ by $x$ $+y$ in (4), we have

$$
f((k+1) x+y)+f((k-1) x-y)=k f(2 x+y)+k f(y)+2 k(k-1) f(x)-2(k-1) f(x+y)
$$

for all $x, y \in X$. Replacing $y$ by $-y$ in (5), we obtain

$$
f((k+1) x-y)+f((k-1) x+y)=k f(2 x-y)+k f(y)+2 k(k-1) f(x)-2(k-1) f(x-y)
$$

for all $x, y \in X$. Adding (5) to (6), we get

$$
\begin{aligned}
f((k+1) x+y)+f((k+1) x-y)+f((k-1) x+y)+f((k-1) x-y) \\
\quad=k[f(2 x+y)+f(2 x-y)]-2(k-1)[f(x+y)+f(x-y)] \\
=-2(k-1)[f(x+y)+f(x-y)]+4 k(k-1) f(x)+2 k f(y)
\end{aligned}
$$

for all $x, y \in X$. From the substitution $y=k x+y$ in (4), we have

$$
\begin{aligned}
f(2 k x+y)+f(y)= & k[f((k+1) x+y)+f((k-1) x+y)] \\
& +2 k(k-1) f(x)-2(k-1) f(k x+y)
\end{aligned}
$$

for all $x, y \in X$. Replacing $y$ by $-y$ in (8), we get

$$
\begin{aligned}
f(2 k x-y)+f(y)= & k[f((k+1) x-y)+f((k-1) x-y)] \\
& +2 k(k-1) f(x)-2(k-1) f(k x-y)
\end{aligned}
$$


for all $x, y \in X$. Adding (8) to (9), we get

$$
\begin{aligned}
f(2 k x+y)+(2 k x-y)=k[ & f((k+1) x+y)+f((k+1) x-y)] \\
& +k[f((k-1) x+y)+f((k-1) x-y)] \\
& -2(k-1)[f(k x+y)+f(k x-y)] \\
& +4 k(k-1) f(x)-2 f(y)
\end{aligned}
$$

for all $x, y \in X$. It follows from (10), by using (4) and (7), that

$$
\begin{aligned}
f(2 k x+y)+f(2 k x-y)=k^{2}[ & f(2 x+y)+f(2 x-y)] \\
& -4 x(k-1)[f(x+y)+f(x-y)] \\
+ & 8 k(k-1) f(x)+2(k-1)(3 k-1) f(y)
\end{aligned}
$$

for all $x, y \in X$. If we replace $x$ by $2 x$ in (4), then we obtain that

$$
\begin{aligned}
f(2 k x+y)+f(2 k x-y)= & k[f(2 x+y)+f(2 x-y)] \\
& +2 k(k-1) f(2 x)-2(k-1) f(y)
\end{aligned}
$$

for all $x, y \in X$. Associating (11) with (12), we conclude that the mapping $f$ satisfies the equation

$$
f(2 x+y)+f(2 x-y)=4[f(x+y)+f(x-y)]+2[f(2 x)-4 f(x)]-6 f(y)
$$

for all $x, y \in X$. Therefore, it follows from Lemma 2.1 that $f$ is quadratic because of the property $f(k x)=k^{2} f(x)$.

Conversely, if a mapping $f$ is quadratic, then it is obvious that $f$ satisfies (4).

\section{Hyers-Ulam stability of (4) in banach spaces}

In this section, let $X$ be a normed space and $Y$ a Banach space. We will investigate the Hyers-Ulam stability problem for the functional equation (4). For notational convenience, we define an operator $D_{k} f(x, y)$ as

$$
\begin{aligned}
D_{k} f(x, y):= & f(k x+y)+f(k x-y)-k f(x+y)-k f(x-y) \\
& -2 k(k-1) f(x)+2(k-1) f(y)
\end{aligned}
$$

for all $x, y \in X$, where $k$ is a fixed integer with $|k|>1$.

Theorem 3.1. Let $\psi: X^{2} \rightarrow[0, \infty)$ be a function such that

$$
\sum_{i=0}^{\infty} \frac{\psi\left(k^{i} x, k^{i} y\right)}{k^{2 i}}<\infty
$$

for all $x, y \in X$. If a mapping $f: X \rightarrow Y$ satisfies the inequality

$$
\left\|D_{k} f(x, y)\right\| \leq \psi(x, y)
$$

for all $x, y \in X$, then there exists a unique quadratic mapping $Q_{1}: X \rightarrow Y$ which satisfies Equation (4) and the inequality

$$
\left\|f(x)-\frac{f(0)}{k+1}-Q_{1}(x)\right\| \leq \frac{1}{2 k^{2}} \sum_{i=0}^{\infty} \frac{\psi\left(k^{i} x, 0\right)}{k^{2 i}}
$$


for all $x \in X$, where $\|f(0)\| \leq \frac{\psi(0,0)}{2 k(k-1)}$. The mapping $Q_{1}$ is defined by

$$
Q_{1}(x)=\lim _{n \rightarrow \infty} \frac{f\left(k^{n} x\right)}{k^{2 n}}
$$

for all $x \in X$.

Proof. Letting $x=y:=0$ in (14), we get

$$
\|f(0)\| \leq \frac{\psi(0,0)}{2 k(k-1)}
$$

Putting $y:=0$ in (14) and dividing by $2 k^{2}$, we obtain

$$
\left\|\frac{f(k x)}{k^{2}}-f(x)+\frac{k-1}{k^{2}} f(0)\right\| \leq \frac{\psi(x, 0)}{2 k^{2}}
$$

for all $x \in X$. Setting $\bar{f}(x):=f(x)-\frac{f(0)}{k+1}$, we lead to a functional inequality

$$
\left\|\frac{\bar{f}(k x)}{k^{2}}-\bar{f}(x)\right\| \leq \frac{\psi(x, 0)}{2 k^{2}}
$$

for all $x \in X$. Using the induction argument on a positive integer $n$ we may obtain that

$$
\left\|\bar{f}(x)-\frac{\bar{f}\left(k^{n} x\right)}{k^{2 n}}\right\| \leq \frac{1}{2 k^{2}} \sum_{i=0}^{n-1} \frac{\psi\left(k^{i} x, 0\right)}{k^{2 i}}
$$

for all $x \in X$. Now, it follows from (19) that for $m>n>0$,

$$
\begin{aligned}
\left\|\frac{\bar{f}\left(k^{m} x\right)}{k^{2 m}}-\frac{\bar{f}\left(k^{n} x\right)}{k^{2 n}}\right\| & =\left\|\frac{\bar{f}\left(k^{m-n+n} x\right)}{k^{2(m-n+n)}}-\frac{\bar{f}\left(k^{n} x\right)}{k^{2 n}}\right\| \\
& =\frac{1}{k^{2 n}}\left\|\bar{f}\left(k^{n} x\right)-\frac{\bar{f}\left(k^{m-n} k^{n} x\right)}{k^{2(m-n)}}\right\| \\
& \leq \frac{1}{2 k^{2}} \sum_{i=0}^{m-n-1} \frac{\psi\left(k^{i+n} x, 0\right)}{k^{2(i+n)}}
\end{aligned}
$$

for all $x \in X$. Since the right-hand side of the inequality (20) tends to 0 as $n \rightarrow \infty$, a sequence $\left\{\frac{\bar{f}\left(k^{n} x\right)}{k^{2 n}}\right\}$ is Cauchy. Therefore, we may define a mapping $Q_{1}: X \rightarrow Y$ as

$$
Q_{1}(x)=\lim _{n \rightarrow \infty} \frac{\bar{f}\left(k^{n} x\right)}{k^{2 n}}=\lim _{n \rightarrow \infty} \frac{f\left(k^{n} x\right)}{k^{2 n}}
$$

for all $x \in X$. Letting $n \rightarrow \infty$ in (19), we lead to the approximation (15).

Next, we have to show that $Q_{1}$ satisfies Equation (4). Replacing $x, y$ by $k^{n} x, k^{n} y$ in (14) and dividing by $k^{2 n}$, we obtain that 


$$
\begin{gathered}
\frac{1}{k^{2 n}} \| f\left(k^{n}(k x+y)\right)+f\left(k^{n}(k x-y)\right)-k f\left(k^{n}(x+y)\right)-k f\left(k^{n}(x-y)\right) \\
-2 k(k-1) f\left(k^{n} x\right)+2(k-1) f\left(x^{n} y\right) \| \leq \frac{1}{k^{2 n}} \psi\left(k^{n} x, k^{n} y\right)
\end{gathered}
$$

for all $x, y \in X$. Taking the limit as $n \rightarrow \infty$, we see from (13) and (16) that the mapping $Q_{1}$ satisfies Equation (4) and so it is quadratic by Theorem 2.2.

To prove the uniqueness of the quadratic mapping $Q_{1}$ satisfying the inequality (15), let us assume that there exists a quadratic mapping $Q_{1}^{\prime}: X \rightarrow Y$ which satisfies the inequality (15). Then, we have $Q_{1}\left(k^{n} x\right)=k^{2 n} \mathrm{Q}_{1}(x)$ and $Q_{1}^{\prime}\left(k^{n} x\right)=k^{2 n} Q_{1}^{\prime}(x)$ for all $x \in$ $X$ and all $n \in \mathrm{N}$. Hence, it follows from (15) that

$$
\begin{aligned}
\left\|Q_{1}(x)-Q_{1}^{\prime}(x)\right\| & =\frac{1}{k^{2 n}}\left\|Q_{1}\left(k^{n} x\right)-Q_{1}^{\prime}\left(k^{n} x\right)\right\| \\
& \leq \frac{1}{k^{2 n}}\left\|Q_{1}\left(k^{n} x\right)-\bar{f}\left(k^{n} x\right)\right\|+\left\|\bar{f}\left(k^{n} x\right)-Q_{1}^{\prime}\left(k^{n} x\right)\right\| \\
& \leq \frac{1}{k^{2}} \sum_{i=n}^{\infty} \frac{\psi\left(k^{i} x, 0\right)}{k^{2 i}}
\end{aligned}
$$

which tends to zero as $n \rightarrow \infty$. This completes the proof of the theorem.

The following theorem is an alternative stability result concerning the stability of functional equation (4).

Theorem 3.2. Let $\psi: X^{2} \rightarrow[0, \infty)$ be a function such that

$$
\sum_{i=1}^{\infty} k^{2 i} \psi\left(\frac{x}{k^{i}}, \frac{y}{k^{i}}\right)<\infty
$$

for all $x, y \in X$. If a mapping $f: X \rightarrow Y$ satisfies the inequality

$$
\left\|D_{k} f(x, y)\right\| \leq \psi(x, y)
$$

for all $x, y \in X$, then there exists a unique quadratic mapping $Q_{2}: X \rightarrow Y$ which satisfies Equation (4) and the inequality

$$
\left\|f(x)-Q_{2}(x)\right\| \leq \frac{1}{2 k^{2}} \sum_{i=1}^{\infty} k^{2 i} \psi\left(\frac{x}{k^{i}}, 0\right)
$$

for all $x \in X$. The mapping $Q_{2}$ is defined by

$$
Q_{2}(x)=\lim _{n \rightarrow \infty} k^{2 n} f\left(\frac{x}{k^{n}}\right)
$$

for all $x \in X$.

Corollary 3.3. Let $\varepsilon_{1} \geq 0, \varepsilon_{2} \geq 0$ and $p, q$ be real numbers such that either $0<p, q<$ 2 or $p, q>2$. If a mapping $f: X \rightarrow Y$ satisfies the inequality

$$
\left\|D_{k} f(x, y)\right\| \leq \varepsilon_{1}\|x\|^{p}+\varepsilon_{2}\|y\|^{q}
$$

for all $x, y \in X$, then there exists a unique quadratic mapping $Q_{i}: X \rightarrow Y(\mathrm{i}=1,2)$ which satisfies (4) and inequality 


$$
\begin{cases}\left\|f(x)-Q_{1}(x)\right\| \leq \frac{\varepsilon_{1}\|x\|^{p}}{2\left(k^{2}-k^{p}\right)}, \text { if } & 0<p, q<2 ; \\ \left\|f(x)-Q_{2}(x)\right\| \leq \frac{\varepsilon_{1}\|x\|^{p}}{2\left(k^{p}-k^{2}\right)}, \text { if } \quad & p, q>2 .\end{cases}
$$

for all $x \in X$. Furthermore, for each fixed $x \in X$ if $f(t x)$ is continuous for all $t \in \mathbf{R}$, then $f(t x)=t^{2} f(x)$ for all $t \in \mathbf{R}$.

Proof. Taking $\psi(x, y)=\varepsilon_{1}\|x\|^{p}+\varepsilon_{2}\|y\|^{q}$ and applying Theorems 3.1 and 3.2, we obtain the desired approximations.

The following is a simple example that the quadratic functional equation $D_{k} f(x, y)=$ $0, k \geq 2$ is not stable for $p=2=q$ in Corollary 3.3.

Example 3.4. Let $\varphi: \mathbf{R} \rightarrow \mathbf{R}$ be defined by

$$
\phi(x)= \begin{cases}\mu x^{2} & \text { if }|x|<1 \\ \mu & \text { otherwise }\end{cases}
$$

where $\mu>0$ is a positive constant, and define $f: \mathbf{R} \rightarrow \mathbf{R}$ by

$$
f(x)=\sum_{i=0}^{\infty} \frac{\phi\left(2^{i} x\right)}{4^{i}}, \quad \text { for all } x \in \mathbf{R} .
$$

Then, $f$ satisfies the functional inequality

$$
\begin{aligned}
& |f(2 x+y)+f(2 x-y) x-2 f(x+y)-2 f(x-y)-4 f(x)+2 f(y)| \\
& \leq 4^{4} \mu\left(|x|^{2}+|y|^{2}\right)
\end{aligned}
$$

for all $x, y \in \mathbf{R}$, but there do not exist a quadratic function $Q: \mathbf{R} \rightarrow \mathbf{R}$ and a constant $\beta>0$ such that

$$
|f(x)-Q(x)| \leq \beta|x|^{2} \text { for all } x \in \mathbf{R} .
$$

Proof. It is easy to see that $f$ is bounded by $\frac{4 \mu}{3}$ on R. If $|x|^{2}+|y|^{2} \geq \frac{1}{4}$ or 0 , then the left side of (25) is less than $16 \mu$, and thus (25) is true. Now suppose that $0<|x|^{2}+|y|^{2}<\frac{1}{4}$. Then there exists a positive integer $k$ such that

$$
\frac{1}{4^{k+2}} \leq|x|^{2}+|y|^{2}<\frac{1}{4^{k+1}}
$$

so that $4^{4}|x|^{2}<\frac{1}{4}, 4^{k}|y|^{2}<\frac{1}{4}$ and $2^{k-1}(2 x \pm y), 2^{k-1}(x \pm y), 2^{k-1} x, 2^{k-1} y$ all belong to the interval $(-1,1)$. Hence, for $i=0,1, \ldots, k-1$,

$$
\phi\left(2^{i}(2 x+y)\right)+\phi\left(2^{i}(2 x-y)\right)-2 \phi\left(2^{i}(x+y)\right)+2 \phi\left(2^{i}(x-y)\right)-4 \phi\left(2^{i} x\right)+2 \phi\left(2^{i} y\right)=0
$$


Therefore, it follows from the definition of $f$ and the inequality (27) that

$$
\begin{aligned}
& |f(2 x+y)+f(2 x-y)-2 f(x+y)-2 f(x-y)-4 f(x)+2 f(y)| \\
& \leq \sum_{i=0}^{\infty} \frac{1}{4^{i}} \mid \phi\left(2^{i}(2 x+y)\right)+\phi\left(2^{i}(2 x-y)\right) \\
& \quad-2 \phi\left(2^{i}(x+y)\right)-2 \phi\left(2^{i}(2 x-y)\right)-4 \phi\left(2^{i} x\right)+2 \phi\left(2^{i} y\right) \mid \\
& \leq \sum_{i=k}^{\infty} \frac{1}{4^{i}} 12 \mu=16 \mu 4^{-k} \leq 4^{4} \mu\left(|x|^{2}+|y|^{2}\right)
\end{aligned}
$$

for all $x, y \in \mathbf{R}$ with $0<|x|^{2}+|y|^{2}<\frac{1}{4}$. Thus $f$ satisfies (25) for all $x, y \in \mathbf{R}$.

We claim that the quadratic functional equation $D_{2} f(x, y)=0$ is not stable for $p=2$ $=q$ in Corollary 3.3. Suppose on the contrary that there exist a quadratic function $Q$ : $\mathbf{R} \rightarrow \mathbf{R}$ and a constant $\beta>0$ satisfying (26). Since $f$ is bounded and continuous for all $x \in \mathbf{R}, Q$ is bounded on any open interval containing the origin and continuous at the origin. Therefore, $Q$ must have the form $Q(x)=\eta x^{2}$ for any $x$ in $\mathbf{R}$. Thus, we obtain that

$$
|f(x)| \leq(\beta+|\eta|)|x|^{2} \quad \text { for all } x \in \mathrm{R} .
$$

However, we can choose a positive integer $m$ with $m \mu>\beta+|\eta|$. If $x \in\left(0, \frac{1}{2^{m-1}}\right)$, then $2^{i} x \in(0,1)$ for all $i=0,1, \ldots, m-1$, and for this $x$ we get

$$
f(x)=\sum_{i=0}^{\infty} \frac{\phi\left(2^{i} x\right)}{4^{n}} \geq \sum_{i=0}^{m-1} \frac{\mu\left(2^{i} x\right)^{2}}{4^{i}}=m \mu x^{2}>(\beta+|\eta|) x^{2},
$$

which contradicts (29). Therefore, the quadratic functional equation $D_{2} f(x, y)=0$ is not stable if $p=2=q$ in Corollary 3.3.

Corollary 3.5. Let $\varepsilon$ be a nonnegative real number. If a mapping $f: X \rightarrow Y$ satisfies the inequality

$$
\left\|D_{k} f(x, y)\right\| \leq \varepsilon
$$

for all $x, y \in X$, then there exists a unique quadratic mapping $Q_{1}: X \rightarrow Y$ which satisfies Equation (4) and the inequality

$$
\left\|f(x)-\frac{f(0)}{k+1}-Q_{1}(x)\right\| \leq \frac{\varepsilon}{2\left(k^{2}-1\right)}
$$

for all $x \in X$. Furthermore, for each fixed $x \in X$ if $f(t x)$ is continuous for all $t \in \mathbf{R}$, then $f(t x)=t^{2} f(x)$ for all $t \in \mathbf{R}$.

Proof. Taking $\psi(x, y)=\varepsilon$ and applying Theorem 3.1, we lead to the approximation.

In the last part, we consider a singular case $k=-1$, which is not investigated in Theorems 3.1 and 3.2 concerning the stability of the functional equation (4).

Theorem 3.6. Let $\psi: X^{2} \rightarrow[0, \infty)$ be a function such that

$$
\sum_{i=0}^{\infty} \frac{\psi\left(2^{i} x, 2^{i} y\right)}{4^{i}}<\infty
$$


for all $x, y \in X$. If a mapping $f: X \rightarrow Y$ satisfies the inequality

$$
\left\|D_{-1} f(x, y)\right\| \leq \psi(x, y)
$$

for all $x, y \in X$, then there exists a unique quadratic mapping $Q_{1}: X \rightarrow Y$ which satisfies the equation $D_{-1} Q_{1}(x, y)=0$ and the inequality

$$
\left\|f(x)-\frac{2 f(0)}{3}-Q_{1}(x)\right\| \leq \frac{1}{8} \sum_{i=0}^{\infty} \frac{\psi\left(2^{i} x, 2^{i} x\right)}{4^{i}}+\frac{1}{4} \sum_{i=0}^{\infty} \frac{\psi\left(2^{i+1} x, 0\right)}{4^{i+1}}
$$

for all $x \in X$, where $\|f(0)\| \leq \frac{\psi(0,0)}{4}$. The mapping $Q_{1}$ is defined by

$$
Q_{1}(x)=\lim _{n \rightarrow \infty} \frac{f\left(2^{n} x\right)}{4^{n}}
$$

for all $x \in X$.

Proof. Letting $x=y:=0$ in (31), we get $\|f(0)\| \leq \frac{\psi(0,0)}{4}$. Putting $y:=0$ in (31) and dividing by 2 , we obtain

$$
\|f(-x)-f(x)-2 f(0)\| \leq \frac{\psi(x, 0)}{2}
$$

for all $x \in X$. Setting $y:=x$ in (31), one has

$$
\|f(-2 x)+f(2 x)-8 f(x)+2 f(0)\| \leq \psi(x, x)
$$

for all $x \in X$. Combining two inequalities above and then letting $\bar{f}(x):=f(x)-\frac{2 f(0)}{3}$, we lead to a functional inequality

$$
\left\|\frac{\bar{f}(2 x)}{4}-\bar{f}(x)\right\| \leq \frac{1}{8} \psi(x, x)+\frac{1}{4^{2}} \psi(2 x, 0)
$$

for all $x \in X$. Using the induction argument on a positive integer $n$, we may obtain that

$$
\left\|\bar{f}(x)-\frac{\bar{f}\left(2^{n} x\right)}{4^{n}}\right\| \leq \frac{1}{8} \sum_{i=0}^{n-1} \frac{\psi\left(2^{i} x, 2^{i} x\right)}{4^{i}}+\frac{1}{4} \sum_{i=0}^{n-1} \frac{\psi\left(2^{i+1} x, 0\right)}{4^{i+1}}
$$

for all $x \in X$. The remaining proof of this theorem follows similarly from the corresponding part of Theorem 3.1.

The following theorem is an alternative stability result concerning the stability of the functional equation (4) with $k=-1$.

Theorem 3.7. Let $\psi: X^{2} \rightarrow[0, \infty)$ be a function such that

$$
\sum_{i=1}^{\infty} 4^{i} \psi\left(\frac{x}{2^{i}}, \frac{y}{2^{i}}\right)<\infty
$$

for all $x, y \in X$. If a mapping $f: X \rightarrow Y$ satisfies the inequality

$$
\left\|D_{-1} f(x, y)\right\| \leq \psi(x, y)
$$


for all $x, y \in X$, then there exists a unique quadratic mapping $Q_{2}: X \rightarrow Y$ which satisfies the equation $D_{-1} Q_{2}(x, y)=0$ and the inequality

$$
\left\|f(x)-Q_{2}(x)\right\| \leq \frac{1}{8} \sum_{i=1}^{\infty} 4^{i} \psi\left(\frac{x}{2^{i}}, \frac{x}{2^{i}}\right)+\frac{1}{4} \sum_{i=1}^{\infty} 4^{i-1} \psi\left(\frac{x}{2^{i-1}}, 0\right)
$$

for all $x \in X$. The mapping $Q_{2}$ is defined by

$$
Q_{2}(x)=\lim _{n \rightarrow \infty} 4^{n} f\left(\frac{x}{2^{n}}\right)
$$

for all $x \in X$.

Corollary 3.8. Let $\varepsilon_{1} \geq 0, \varepsilon_{2} \geq 0$ and $p, q$ be real numbers such that either $0<p, q<$ 2 or $p, q>2$. If a mapping $f: X \rightarrow Y$ satisfies the inequality

$$
\left\|D_{-1} f(x, y)\right\| \leq \varepsilon_{1}\|x\|^{p}+\varepsilon_{2}\|y\|^{q}
$$

for all $x, y \in X$, then there exists a unique quadratic mapping $Q_{i}: X \rightarrow Y(\mathrm{i}=1,2)$ which satisfies the equation $D_{-1} Q_{i}(x, y)=0$ and inequality

$$
\begin{cases}\left\|f(x)-Q_{1}(x)\right\| \leq \frac{\varepsilon_{1}\left(2+2^{p}\right)\|x\|^{p}}{4\left(2^{2}-2^{p}\right)}+\frac{\varepsilon_{2}\|x\|^{q}}{2\left(2^{2}-2^{q}\right)}, \text { if } & 0<p, q<2 ; \\ \left\|f(x)-Q_{2}(x)\right\| \leq \frac{\varepsilon_{1}\left(2+2^{p}\right)\|x\|^{p}}{4\left(2^{p}-2^{2}\right)}+\frac{\varepsilon_{2}\|x\|^{q}}{2\left(2^{q}-2^{2}\right)}, \text { if } \quad p, q>2 .\end{cases}
$$

for all $x \in X$. Furthermore, for each fixed $x \in X$ if $f(t x)$ is continuous for all $t \in \mathbf{R}$, then $f(t x)=t^{2} f(x)$ for all $t \in \mathbf{R}$.

\section{Hyers-Ulam stability of (4) in non-archimedean spaces}

In this section, let $X$ be a vector space and $Y$ a complete non-Archimedean space. We recall that a field $\mathbf{K}$, equipped with a function (non-Archimedean absolute value, valuation) $|\cdot|$ from $\mathbf{K}$ into $[0, \infty)$, is called a non-Archimedean field if the function $|\cdot|: \mathbf{K}$ $\rightarrow[0, \infty)$ satisfies the following conditions:

(1) $|r|=0$ if and only if $r=0$;

(2) $|r s|=|r||s|$;

(3) the strong triangle inequality, namely, $|r+s| \leq \max \{|r|,|s|\}$ for all $r, s \in \mathbf{K}$.

Clearly, $|1|=1=|-1|$ and $|n| \leq 1$ for all nonzero integer $n$.

Let $Y$ be a vector space over the non-Archimedean field $\mathbf{K}$ with a non-trivial nonArchimedean valuation $|\cdot|$. A function $\|\cdot\|: Y \rightarrow[0, \infty)$ is called a non-Archimedean norm (valuation) if it satisfies the following conditions:

(1) $\|x\|=0$ if and only if $x=0$;

(2) $|r x|=|r|\|x\|$ for all $x \in Y$ and all $r \in \mathbf{K}$;

(3) the strong triangle inequality, namely,

$$
\|x+y\| \leq \max \{\|x\|,\|y\|\}
$$

for all $x, y \in Y$. 
In this case, the pair $(Y,\|\cdot\|)$ is called a non-Archimedean space. By a complete non-Archimedean space we mean one in which every Cauchy sequence is convergent. It follows from the strong triangle inequality that

$$
\left\|x_{n}-x_{m}\right\| \leq \max \left\{\left\|x_{j+1}-x_{j}\right\|: m \leq j<n-1\right\}
$$

for all $x_{n}, x_{m} \in Y$ and all $m, n \in \mathbf{N}$ with $n>m$. Therefore, a sequence $\left\{x_{n}\right\}$ is a Cauchy sequence in non-Archimedean space $(Y,\|\cdot\|)$ if and only if the sequence $\left\{x_{n+1}-x_{n}\right\}$ converges to zero in the space $(Y,\|\cdot\|)$. Now, we will investigate the generalized the Hyers-Ulam stability problem for the functional equation (4) in a complete non-Archimedean space $Y$.

Theorem 4.1. Let $\psi: X^{2} \rightarrow[0, \infty)$ be a function such that

$$
\begin{gathered}
\Psi_{1}(x):=\lim _{n \rightarrow \infty} \max \left\{\frac{\psi\left(k^{i} x, 0\right)}{|k|^{2 i}}: 0 \leq i<n\right\}<\infty, \\
\lim _{n \rightarrow \infty} \frac{\psi\left(k^{n} x, k^{n} y\right)}{|k|^{2 n}}=0
\end{gathered}
$$

for all $x, y \in X$. If a mapping $f: X \rightarrow Y$ satisfies the inequality

$$
\left\|D_{k} f(x, y)\right\| \leq \psi(x, y)
$$

for all $x, y \in X$, then there exists a quadratic mapping $Q_{1}: X \rightarrow Y$ which satisfies Equation (4) and the inequality

$$
\left\|f(x)-Q_{1}(x)\right\| \leq \frac{1}{|2||k|^{2}} \Psi_{1}(x)
$$

for all $x \in X$, where $\|f(0)\| \leq \frac{\psi(0,0)}{|2 k(k-1)|}$. The mapping $Q_{1}$ is defined by

$$
Q_{1}(x)=\lim _{n \rightarrow \infty} \frac{f\left(k^{n} x\right)}{k^{2 n}}
$$

for all $x \in X$. Moreover, if

$$
\lim _{l \rightarrow \infty} \frac{\Psi_{1}\left(k^{l} x\right)}{|k|^{2 l}}=\lim _{l \rightarrow \infty} \lim _{n \rightarrow \infty} \max \left\{\frac{\psi\left(k^{j} x, 0\right)}{|k|^{2 j}}: l \leq j<l+n\right\}=0
$$

for all $x \in X$, then $Q_{1}$ is a unique quadratic mapping satisfying (42).

Proof. Letting $x=y:=0$ in (41), we get $f(0)=0$ because $\psi(0,0)=0$ by the condition (40). Putting $y:=0$ in (41) and dividing by $2|k|^{2}$, we obtain

$$
\left\|\frac{f(k x)}{k^{2}}-f(x)\right\| \leq \frac{\psi(x, 0)}{2|k|^{2}}
$$

for all $x \in X$, where $|k| \leq 1$ is a non-Archimedean valuation. Replacing $x$ by $k^{n} x$ in (44) and dividing by $|k|^{2 n}$,

$$
\left\|\frac{f\left(k^{n+1} x\right)}{k^{2 n+2}}-\frac{f\left(k^{n} x\right)}{k^{2 n}}\right\| \leq \frac{\psi\left(k^{n} x, 0\right)}{2|k|^{2 n+2}}
$$

for all $x \in X$. Since the right-hand side of the inequality (45) tends to 0 as $n \rightarrow \infty$, a sequence $\left\{\frac{f\left(k^{n} x\right)}{k^{2 n}}\right\}$ is Cauchy in the complete non-Archimedean space $Y$. Therefore, we 
may define a mapping $Q_{1}: X \rightarrow Y$ as

$$
Q_{1}(x)=\lim _{n \rightarrow \infty} \frac{f\left(k^{n} x\right)}{k^{2 n}}
$$

for all $x \in X$. Using the induction argument and the strong triangle inequality, we may obtain that

$$
\left\|f(x)-\frac{f\left(k^{n} x\right)}{k^{2 n}}\right\| \leq \frac{1}{2|k|^{2}} \max \left\{\frac{\psi\left(k^{i} x, 0\right)}{|k|^{2 i}}: 0 \leq i<n\right\}
$$

for all $x \in X$. Letting $n \rightarrow \infty$ in (46), we lead to the approximation (42).

Next, we have to show that $Q_{1}$ satisfies Equation (4). Replacing $x, y$ by $k^{n} x, k^{n} y$ in (41) and dividing by $|k|^{2 n}$, it then follows that

$$
\begin{gathered}
\frac{1}{|k|^{2 n}} \| f\left(k^{n}(k x+y)\right)+f\left(k^{n}(k x-y)\right)-k f\left(k^{n}(x+y)\right)-k f\left(k^{n}(x-y)\right) \\
-2 k(k-1) f\left(k^{n} x\right)+2(k-1) f\left(k^{n} y\right) \| \leq \frac{1}{|k|^{2 n}} \psi\left(k^{n} x, k^{n} y\right)
\end{gathered}
$$

for all $x, y \in X$. Taking the limit as $n \rightarrow \infty$, we see from (40) and (43) that

$$
\begin{aligned}
& Q_{1}(k x+y)+Q_{1}(k x-y) \\
& =k Q_{1}(x+y)+k Q_{1}(x-y)+2 k(k-1) Q_{1}(x)-2(k-1) Q_{1}(y)
\end{aligned}
$$

for all $x, y \in X$. Therefore, the mapping $Q_{1}$ satisfies Equation (4) and so it is quadratic by Theorem 2.2 .

Moreover, to prove the uniqueness of the quadratic mapping $Q_{1}$ satisfying the inequality (42), let us assume that there exists a quadratic mapping $Q_{1}^{\prime}:=X \rightarrow Y$ which satisfies the inequality (42). Then, we have $Q_{1}\left(k^{l} x\right)=k^{2 l} Q_{1}(x)$ and $Q_{1}^{\prime}\left(k^{l} x\right)=k^{2 l} Q_{1}^{\prime}(x)$ for all $x \in X$ and all $l \in \mathrm{N}$. Hence, it follows from (42) that for all $x$ $\in X$

$$
\begin{aligned}
\left\|Q_{1}(x)-Q_{1}^{\prime}(x)\right\| & =\frac{1}{|k|^{2 l}}\left\|Q_{1}\left(k^{l} x\right)-Q_{1}^{\prime}\left(k^{l} x\right)\right\| \\
& \leq \frac{1}{|k|^{2 l}} \max \left\{\left\|Q_{1}\left(k^{l} x\right)-f\left(k^{l} x\right)\right\|,\left\|f\left(k^{l} x\right)-Q_{1}^{\prime}\left(k^{l} x\right)\right\|\right\} \\
& \leq \frac{1}{|k|^{2}} \lim _{n \rightarrow \infty} \max \left\{\frac{\psi\left(k^{l+i} x, 0\right)}{|k|^{2(l+1)}}: 0 \leq i<n\right\} \\
& =\frac{1}{|k|^{2}} \lim _{n \rightarrow \infty} \max \left\{\frac{\psi\left(k^{j} x, 0\right)}{|k|^{2 j}}: l \leq j<l+n\right\} \\
& =\frac{1}{|k|^{2}} \frac{\Psi_{1}\left(k^{l} x\right)}{|k|^{2 l}}, \forall l \in \mathrm{N},
\end{aligned}
$$

which tends to zero as $l \rightarrow \infty$. This completes the proof of the theorem.

Theorem 4.2. Let $\psi: X^{2} \rightarrow[0, \infty)$ be a function such that

$$
\begin{array}{r}
\Psi_{2}(x):=\lim _{n \rightarrow \infty} \max \left\{|k|^{2 i} \psi\left(\frac{x}{k^{i}}, 0\right): 1 \leq i \leq n\right\}<\infty \\
\lim _{n \rightarrow \infty}|k|^{2 n} \psi\left(\frac{x}{k^{n}}, \frac{y}{k^{n}}\right)=0
\end{array}
$$


for all $x, y \in X$. If a mapping $f: X \rightarrow Y$ satisfies the inequality

$$
\left\|D_{k} f(x, y)\right\| \leq \psi(x, y)
$$

for all $x, y \in X$, then there exists a quadratic mapping $Q_{2}: X \rightarrow Y$ which satisfies Equation (4) and the inequality

$$
\left\|f(x)-\frac{f(0)}{k+1}-Q_{2}(x)\right\| \leq \frac{1}{|2||k|^{2}} \Psi_{2}(x)
$$

for all $x \in X$, where $\|f(0)\| \leq \frac{\psi(0,0)}{|2 k(k-1)|}$. The mapping $Q_{2}$ is defined by

$$
Q_{2}(x)=\lim _{n \rightarrow \infty} k^{2 n} f\left(\frac{x}{k^{n}}\right)
$$

for all $x \in X$. Moreover, if $\lim _{l \rightarrow \infty}|k|^{2 l} \Psi_{2}\left(\frac{x}{k^{l}}\right)=0$ for all $x \in X$, then $Q_{2}$ is a unique quadratic mapping satisfying (49).

Proof. Letting $x=y:=0$ in (48), we get

$$
\|f(0)\| \leq \frac{\psi(0,0)}{|2 k(k-1)|}
$$

where $|2 k(k-1)| \leq 1$ is a non-Archimedean valuation. Putting $y=0$ in (48), one has

$$
\begin{aligned}
&|2|\left\|f(k x)-k^{2} f(x)+(k-1) f(0)\right\| \leq \psi(x, 0), \\
& \text { i.e., }\left\|\bar{f}(x)-k^{2} \bar{f}\left(\frac{x}{k}\right)\right\| \leq \frac{1}{|2||k|^{2}}|k|^{2} \psi\left(\frac{x}{k^{\prime}}, 0\right)
\end{aligned}
$$

for all $x \in X$, where $\bar{f}(x)=f(x)-\frac{f(0)}{k+1}$. Replacing $x$ by $\frac{x}{k^{n}}$ in (51) and multiplying $|k|$ ${ }^{2 n}$, we have

$$
\left\|k^{2 n} f\left(\frac{x}{k^{n}}\right)-k^{2 n+2} f\left(\frac{x}{k^{n+1}}\right)\right\| \leq \frac{1}{|2|}|k|^{2 n+2} \psi\left(\frac{x}{k^{n+1}}, 0\right)
$$

for all $x \in X$. Since the right-hand side of the inequality (52) tends to 0 as $n \rightarrow \infty$, the sequence $\left\{k^{2 n} f\left(\frac{x}{k^{n}}\right)\right\}$ is Cauchy. Therefore, one may define a mapping $Q_{2}: X \rightarrow Y$ by

$$
Q_{2}(x)=\lim _{n \rightarrow \infty} k^{2 n} f\left(\frac{x}{k^{n}}\right)
$$

for all $x \in X$. Using induction on positive integers $n$, we obtain that

$$
\left\|f(x)-k^{2 n} f\left(\frac{x}{k^{n}}\right)\right\| \leq \frac{1}{|2||k|^{2}} \max \left\{|k|^{2 i} \psi\left(\frac{x}{k^{i}}, 0\right): 1 \leq i \leq n\right\}
$$

for all $x \in X$. Letting $n \rightarrow \infty$ in (53), we arrive at the estimation (49).

The remaining assertion is similar to that of Theorem 4.1.

Corollary 4.3. Let $\varepsilon_{1} \geq 0, \varepsilon_{2} \geq 0$ and $p, q$ be real numbers such that either $0<p, q<$ 2 or $p, q>2$. If a mapping $f: X \rightarrow Y$ satisfies the inequality

$$
\left\|D_{k} f(x, y)\right\| \leq \varepsilon_{1}\|x\|^{p}+\varepsilon_{2}\|y\|^{q}
$$


for all $x, y \in X$, then there exists a unique quadratic mapping $Q_{i}: X \rightarrow Y(\mathrm{i}=1,2)$ which satisfies (4) and inequality

$$
\begin{cases}\left\|f(x)-Q_{1}(x)\right\| \leq \frac{\varepsilon_{1}\|x\|^{p}}{|2||k|^{2}} \leq \frac{\varepsilon_{1}\|x\|^{p}}{|2||k|^{p}}, \quad \text { if } \quad p, q>2 ; \\ \left\|f(x)-\frac{f(0)}{k+1}-Q_{2}(x)\right\| \leq \frac{\varepsilon_{1}\|x\|^{p}}{|2||k|^{p}} \leq \frac{\varepsilon_{1}\|x\|^{p}}{|2||k|^{2}}, \quad \text { if } \quad 0<p, q<2 .\end{cases}
$$

for all $x \in X$.

Proof. Taking $\psi(x, y)=\varepsilon_{1}\|x\|^{p}+\varepsilon_{2}\|y\|^{q}$ and applying Theorems 4.1 and 4.2, we obtain the desired approximations.

Corollary 4.4. Let $\varepsilon$ be a nonnegative real number. If a mapping $f: X \rightarrow Y$ satisfies the inequality

$$
\left\|D_{k} f(x, y)\right\| \leq \varepsilon
$$

for all $x, y \in X$, then there exists a unique quadratic mapping $Q_{1}: X \rightarrow Y$ which satisfies Equation (4) and the inequality

$$
\left\|f(x)-\frac{f(0)}{k+1}-Q_{1}(x)\right\| \leq \frac{\varepsilon}{|2|}
$$

for all $x \in X$.

We remark that stability results of the Euler-Lagrange-type quadratic equation (4) in normed spaces are very different from those of Equation (4) in non-Archimedean normed spaces, of which stability results in non-Archimedean normed spaces maybe come from the opposite direction to stability results in normed spaces in view of Corollaries 4.3 and 4.4.

\section{Acknowledgements}

The authors would like to thank the referees and the editors for carefully reading this article and for their valuable comments. This study was supported by the Basic Research Program through the National Research Foundation of Korea funded by the Ministry of Education, Science and Technology (No. 2011-0002614).

\section{Authors' contributions}

All authors carried out the proof. All authors conceived of the study, and participated in its design and coordination. All authors read and approved the final manuscript.

\section{Competing interests}

The authors declare that they have no competing interests.

Received: 18 April 2011 Accepted: 7 March 2012 Published: 7 March 2012

\section{References}

1. Ulam, SM: A Collection of Mathematical Problems. Interscience Publication, New York (1960)

2. Hyers, DH: On the stability of the linear functional equation. Proc Natl Acad Sci. 27, 222-224 (1941). doi:10.1073/ pnas.27.4.222

3. Aoki, T: On the stability of the linear transformation in Banach spaces. J Math Soc Jpn. 2, 64-66 (1950). doi:10.2969/ jmsj/00210064

4. Rassias, TM: On the stability of the linear mapping in Banach spaces. Proc Am Math Soc. 72, 297-300 (1978). doi:10.1090/S0002-9939-1978-0507327-1

5. Forti, GL: Hyers-Ulam stability of functional equations in several variables. Aequationes Math. 50, $143-190$ (1995). doi:10.1007/BF01831117

6. Găvruta, P: A generalization of the Hyers-Ulam-Rassias stability of approximately additive mappings. J Math Anal Appl. 184, 431-436 (1994). doi:10.1006/jmaa.1994.1211

7. Jun, K, Lee, Y: On the Hyers-Ulam-Rassias stability of a pexiderized quadratic inequality. Math Inequal Appl. 4(1):93-118 (2001)

8. Kannappan, P: Quadratic functional equation and inner product spaces. Results Math. 27, 365-372 (1995)

9. Skof, F: Local properties and approximations of operators (Italian). Rend Sem Mat Fis Milano. 53, 113-129 (1983). doi:10.1007/BF02924890 
10. Cholewa, PW: Remark on the stability of functional equations. Aequationes Math. 27, 76-86 (1984). doi:10.1007/ BF02192660

11. Czerwik, S: On the stability of the quadratic mapping in normed spaces. Abh Math Sem Univ Hamburg. 62, 59-64 (1992). doi:10.1007/BF02941618

12. Rassias, TM: On the stability of functional equations in Banach spaces. J Math Anal Appl. 251, 264-284 (2000) doi:10.1006/jmaa.2000.7046

13. Borelli, C, Forti, GL: On a general Hyers-Ulam stability. Int J Math Sci. 18, 229-236 (1995). doi:10.1155/ S0161171295000287

14. Bae, J, Jun, K: On the Hyers-Ulam-Rassias stability of a quadratic functional equation. Bull Korean Math Soc. 38(2):325-336 (2001)

15. Eshaghi Gordji, M, Khodaei, $\mathrm{H}$ : On the generalized Hyers-Ulam-Rassias stability of quadratic functional equations. Abstr Appl Anal 2009 (2009). Article ID 923476, 11

16. Eshaghi Gordji, M, Khodabakhsh, R, Jung, S, Khodaei, H: AQCQ-functional equation in non-Archimedean normed spaces. Abstr Appl Anal 2010 (2010). Article ID 741942, 22

17. Eshaghi Gordji, M, Khodaei, H, Kim, H: Approximate quartic and quadratic mappings in quasi-Banach spaces. Int J Math Math Sci 2011 (2011). Article ID 734567, 18

18. Jung, S: On the Hyers-Ulam stability of the functional equations that have the quadratic property. J Math Anal Appl. 222, 126-137 (1998). do:10.1006/jmaa.1998.5916

19. Jung, S: On the Hyers-Ulam-Rassias stability of a quadratic functional equation. J Math Anal Appl. 232, 384-393 (1999). doi:10.1006/jmaa.1999.6282

20. Jun, K, Kim, H: Remark on the stability of functional equation. Bull Korean Math Soc 38(2):679-687 (2001). 12

21. Rassias, JM: On the stability of the Euler-Lagrange functional equation. Chin J Math. 20, 185-190 (1992)

22. Rassias, JM: On the stability of the non-linear Euler-Lagrange functional equation in real normed linear spaces. J Math Phys Sci. 28, 231-235 (1994)

23. Rassias, JM: On the stability of the general Euler-Lagrange functional equation. emonstratio Math. 29, 755-766 (1996)

24. Ravi, K, Murali, R, Arunkumar, M: The generalized Hyers-Ulam-Rassias stability of a quadratic functional equation. J Inequal Pure Appl Math 9(1) (2008). Article ID 20

25. Gordji, M, Savadkouhi, M, Park, C: Quadratic-quartic functional equations in RN-spaces. J Inequal Appl 2009 (2009). Article ID 868423, 14

26. Gordji, M, Abbaszadeh, S, Park, C: On the stability of a generalized quadratic and quartic type functional equation in quasi-Banach spaces. J Inequal Appl 2009 (2009). Article ID 153084, 26

27. Kim, H: On the stability problem for a mixed type of quartic and quadratic functional equation. J Math Anal Appl. 324, 358-372 (2006). doi:10.1016/j.jmaa.2005.11.053

doi:10.1186/1029-242X-2012-58

Cite this article as: Kim et al:: Approximate Euler-Lagrange quadratic mappings. Journal of Inequalities and Applications 2012 2012:58.

\section{Submit your manuscript to a SpringerOpen ${ }^{\odot}$} journal and benefit from:

- Convenient online submission

- Rigorous peer review

- Immediate publication on acceptance

- Open access: articles freely available online

- High visibility within the field

- Retaining the copyright to your article

Submit your next manuscript at $\boldsymbol{\wedge}$ springeropen.com 